\title{
EDITORIAL
}

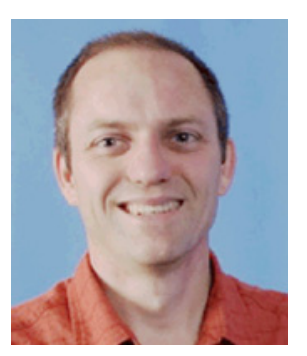

\section{Future of Lab on a Chip}

David J. Beebe

(C) The Korean Society of Medical \& Biological Engineering and Springer 2012

Biological and medical applications of lab a on a chip is a timely topic that is active area of research worldwide. Biomedical Engineering Letters is well positioned to contribute to reporting important results in this area. The field is continually evolving as lab on chip technologies and associated fabrication methods mature and the focus of research turns more and more towards the applications. A recent example is the application of lab on a chip to tackle the problem of the capture and analysis of circulating tumor cells (CTCs). CTCs are rare cells (approximately 1 in a billion) that have the potential to improve patient diagnosis, monitoring and response to treatment. Another example is the application of lab on a chip the measurement of immune cell response which may lead to improved asthma diagnosis as well as improving our understanding of the mechanisms that regulated directed cell migration. The development of lab on a chip for global health and point of care diagnostics are other emerging application areas. Encouragingly, the focus of much current work has moved beyond technology development and demonstration and into clinical use.

These are examples that provide appropriate matches between the problem and the technology. The ability of lab on a chip to control the microenvironment (e.g. create stable biochemical gradients) opens up new avenues of biological inquiry and the potential for more personalized diagnosis and treatment. Lab on a chip can enable "more with less" which will enable the use of small numbers of primary cells from biopsies for companion diagnostics. The forces dominant at the microscale provide unique capabilities that are now being applied in practical ways to address challenges in medicine and biology such as single cell analysis and biomolecular sensing. Such advances allow new lines of inquiry and discovery as well as higher resolution measurements. The future is bright at the interface between lab on a chip and biology/medicine. This issue contains many examples of progress towards increased understanding of biology and improved patient care. I look forward to BMEL's continued leadership in bringing the latest updates in lab a chip to a broad audience. In closing, I am pleased to serve on the editorial board of Biomedical Engineering Letters and to have the opportunity to contribute this editorial to this issue that is focused on lab on a chip.

David J. Beebe $(\square)$

Department of Biomedical Engineering, University of Wisconsin-Madison, Rm 6009, Wisconsin Institute for Medical Research, 1111 Highland Drive, Madison, WI 53705

Tel : +608-262-2260 / Fax : +608-265-6905

E-mail : djbeebe@wisc.edu 